\title{
Production of Free Radicals from Adamantane by High Energy Irradiation
}

\author{
W. G. FilbY and K. GüNTHER \\ Institut für Radiochemie, Kernforschungszentrum Karlsruhe, Karlsruhe, W.-Germany
}

(Z. Naturforsch. 27 b, 1289-1293 [1972] ; received July 28, 1972)

Nature and stability of the radicals, independence of radiation quality, mechanism of formation of trapped radicals

\begin{abstract}
The nature and properties of radicals produced by the action of electron and $\gamma$-radiation on adamantane have been investigated. Radicals produced by irradiation at $77^{\circ} \mathrm{K}$ are stable to around room temperature, while irradiation at the latter temperature yields well resolved spectra of species stable to about $130{ }^{\circ} \mathrm{C}$. The significance of the latter, assigned as the 1-adamantyl radical, in the formation of trapped radicals is discussed.
\end{abstract}

The hydrocarbon adamantane, tricyclo $\left(3,3,1,1^{3,7}\right)$ decane shows some unusual properties. An easily sublimed solid at room temperature its melting point is $270{ }^{\circ} \mathrm{C}$ in a sealed tube ${ }^{*}$. The low temperature form undergoes a phase transition at $-65^{\circ} \mathrm{C}$ involving a change from a tetragonal structure to a face centred cubic one ${ }^{1}$. NMR experiments can only by interpreted on the assumption that in the latter form free rotation of the molecules can occur ${ }^{2}$. Despite its nearly tetrahedral angles and the staggered conformation of adjacent carbon atoms, thermochemical data show it indeed not to be strain free.

Several recent reports have been concerned with the production of free radicals from adamantane ${ }^{3,4}$ and in adamantane used as an "inert" matrix for unstable species not normally accessible to ESR investigation ${ }^{5-7}$. GEE et al. ${ }^{3}$ studying the action of $\gamma$-rays on solid adamantane, concluded that the dominant paramagnetic species produced at $77^{\circ} \mathrm{K}$ is the 2-adamantyl radical. This interpretation has been questioned by FerRell et al. ${ }^{4}$, however who re-examined the problem with a more carefully purified sample. X-irradiation at $77^{\circ} \mathrm{K}$ yielded a spectrum which was again assigned to 2-adamantyl. No evidence however was found for the participation of the 1-adamantyl radical despite similar experimental conditions.

MARX and Bonnazola ${ }^{14}$ have recently offered a completely different explanation based on a pro-

Requests for reprints should be sent to Dr. W. G. Filby, Kernforschungszentrum Karlsruhe, Institut für Radiochemie, D-7500 Karlsruhe, Postfach 3640.

* $n$-decane melts at $-31^{\circ} \mathrm{C}$. posed fission of the primary adamantane cation yielding the 1 methyl 1 cyclohexenyl radical (1).<smiles>C1=CC2CCC=C1C2</smiles>

We have already commented on these results ${ }^{9 a, b}$.

A study of the radiation chemistry of adamantane was undertaken by HATTENBACH and VOM BAUR ${ }^{8}$ who observed, after $\gamma$-irradiation at $300{ }^{\circ} \mathrm{C}$, a $\mathrm{G}$ (adamantane) value of 3.0 and gaseous products consisting chiefly of $\mathrm{Cl}-\mathrm{C} 3$ hydrocarbons and hydrogen.

With the goal of establishing a) the nature and properties of free radicals produced in irradiated pure adamantane, $b$ ) their dependence on the quality of the radiation, c) the mechanism of formation of radicals produced from guest molecule trapped in an adamantane matrix, we undertook the work described below.

\section{Experimental}

\section{Materials}

All samples of adamantane (Fluka) used in this study were purified in four stages in the following manner: i) Double recrystallization from hot hexane containing active carbon followed by vacuum drying. The product showed traces of six different compounds amounting to a total concentration of foreign material of greater than $1 \%$.

ii) Microsublimation under reduced pressure. The gas chromatogram of the resulting product still revealed the presence of two foreign substances whose peaks were placed symmetrically about that of adamantane. The concentration of impurity remained at about $0.05 \%$. 
iii) Large scale zone refining. Batches of adamantane $(20 \mathrm{~g})$ were loaded into a Desaga DBGM zone refining apparatus. In all, this purification stage consisted of traversal of $72 \times 1 \mathrm{~cm}$ zones at $2 \mathrm{~mm} / \mathrm{h}$. The resulting adamantane, taken from the top of the zone refining tube showed no impurity peaks in the gas chromatogram. The lower limit of our detectability is around $20 \mathrm{ppm}$.

iv) Small scale zone refining and ESR sample preparation.

The purest adamantane from stage iii) was homogenised, loaded into tubes of Schott radiation resistant glass and sealed off. Two such tubes were then bound together and zone refined for a further 72 zones. The resulting material was then used directly for irradiation and ESR measurements. The resulting material was then used directly for irradiation and ESR measurments. The process was repeated in order to provide material for all four experiments. Gas chromatography of these samples was naturally impossible, however care was taken in the ESR measurements to exclude the lower, possibly impurer part of the refined column from the ESR cavity.

\section{Gas chromatography}

All gas chromatographic analyses were conducted on a Varian 1960 gas chromatograph fitted with a flame ionisation detector. The operating conditions were as follows, column: $6^{\prime} \times 1 / 4^{\prime \prime}$ glass column containing $5 \%$ FFAP on Chromosorb G run at $100^{\circ} \mathrm{C}$, carrier gas: nitrogen at a flow rate of $30 \mathrm{ml} / \mathrm{min}$. Injections were performed in hexane solution. Under these conditions the retention time of adamantane was approximately $10 \mathrm{~min}$.

\section{Irradiation facilities}

All $\gamma$-irradiations were carried out using an AEG Gammacell 220. The observed dose rate, determined by the Fricke method, was $1.65 \times 10^{19} \mathrm{eV} / \mathrm{g} / \mathrm{h}$. The linac irradiations were performed on a Varian type V 7703 electron linear accelerator, providing an electron energy of $10 \mathrm{MeV}$.

\section{ESR measurements}

The ESR spectrometer used in this work was an AEG 20X fitted with $20 \mathrm{~cm}$ magnet and $100 \mathrm{kc} / \mathrm{s}$ modulation. Magnetic field sweep calibration was conducted with an AEG nuclear magnetic resonance fieldmeter using potassium nitrosodisulphonate (13.0 gauss) as primary standard. Microwave frequency measurements were performed with a Hewlett-Packard 52AG-L Electronic Counter.

The temperature was maintained either by standing the sample tube in liquid nitrogen in a quartz dewar vessel or by use of a variable temperature unit supplied by AEG Ltd. The sample temperature was monitored with a copper-constantan thermocouple.

\section{Results and Discussion}

The nature and temperature dependence of the spectra

Fig. 1 shows the ESR spectra, recorded at several temperature of a $\gamma$ (a) and electron irradiated (b) adamantane rod. They are typical ESR spectra of radicals observed at low temperature in the solid
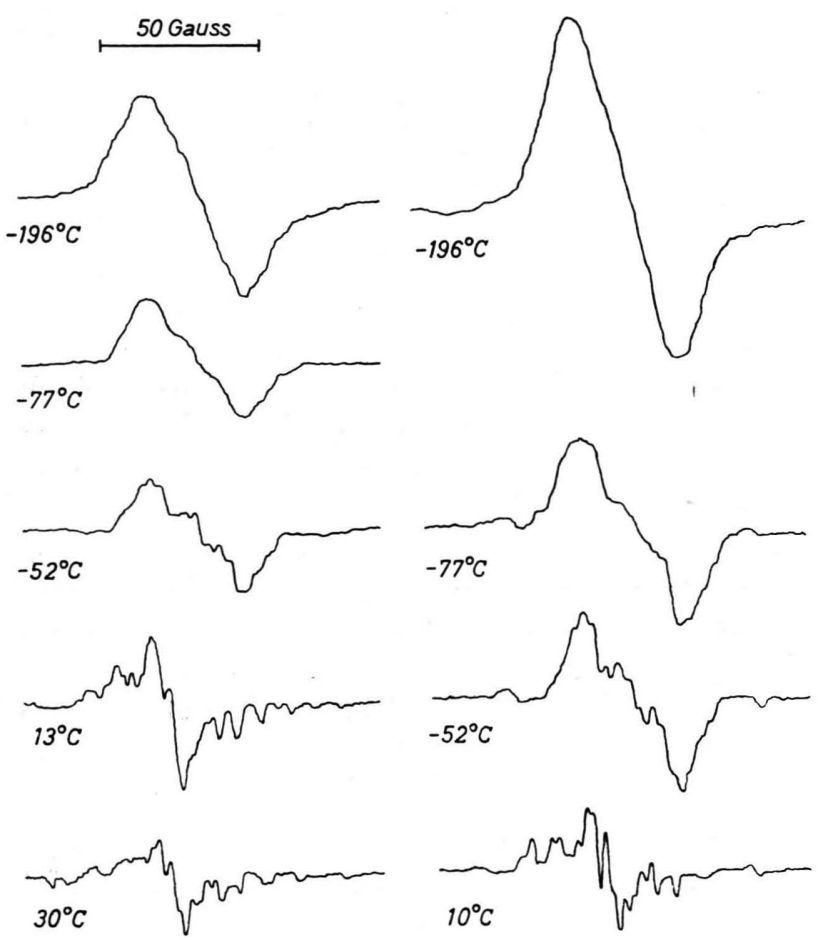

a)

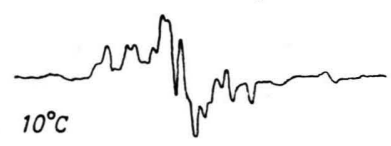

b)

Fig. l. ESR spectra of a) $\mathrm{Co}^{60} \gamma$ and b) electron irradiated adamantane. Irradiation temperature $-196^{\circ} \mathrm{C}$. Dose $=$ 0.5 Mrad.

phase and at $77^{\circ} \mathrm{K}$ consist of a broad singlet of peat-to peak width -43 gauss. While the signals observed in the temperature range $77^{\circ}-200^{\circ} \mathrm{K}$ were stable and could be kept for days with no change in spectral form and only little reduction in intensity, at higher temperatures the behaviour altered. The spectra became narrower and better resolved and the decay rate considerably faster, until at $30^{\circ} \mathrm{C}$ an ESR signal was only just detectable.

Fig. 2 shows the ESR spectra obtained from samples irradiated at $25^{\circ}$ with a) $\gamma$-rays and b) electrons respectively. Clearly the quality of the irradiation is without effect in controlling the nature 

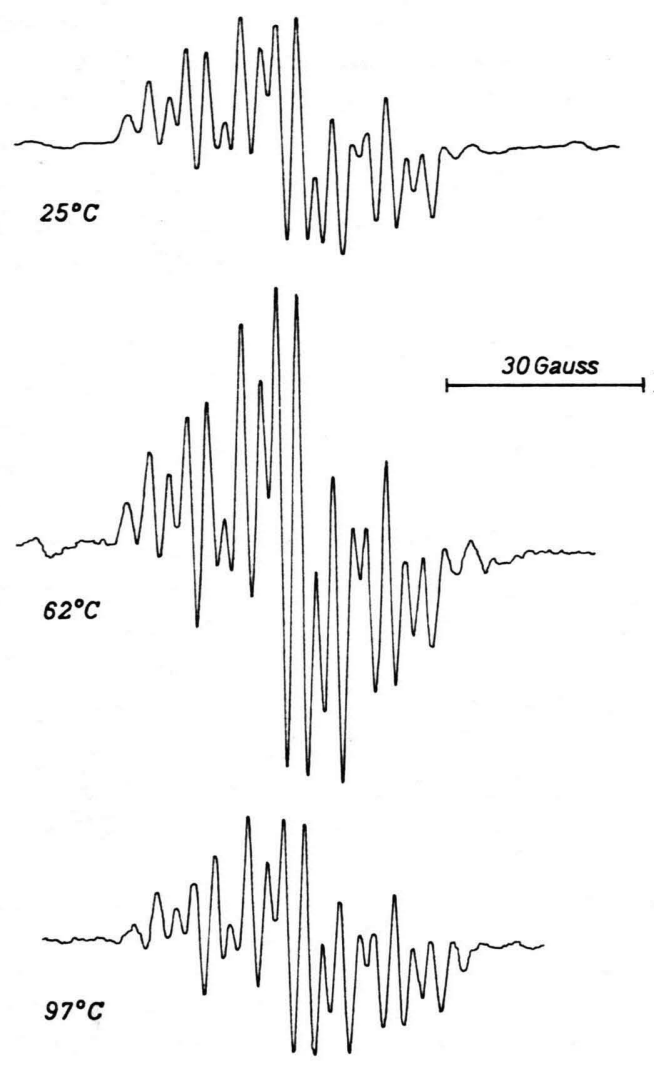

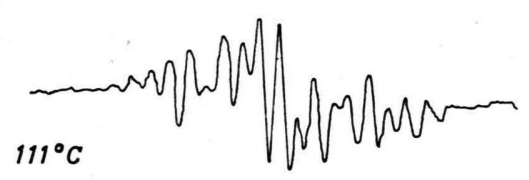

a)
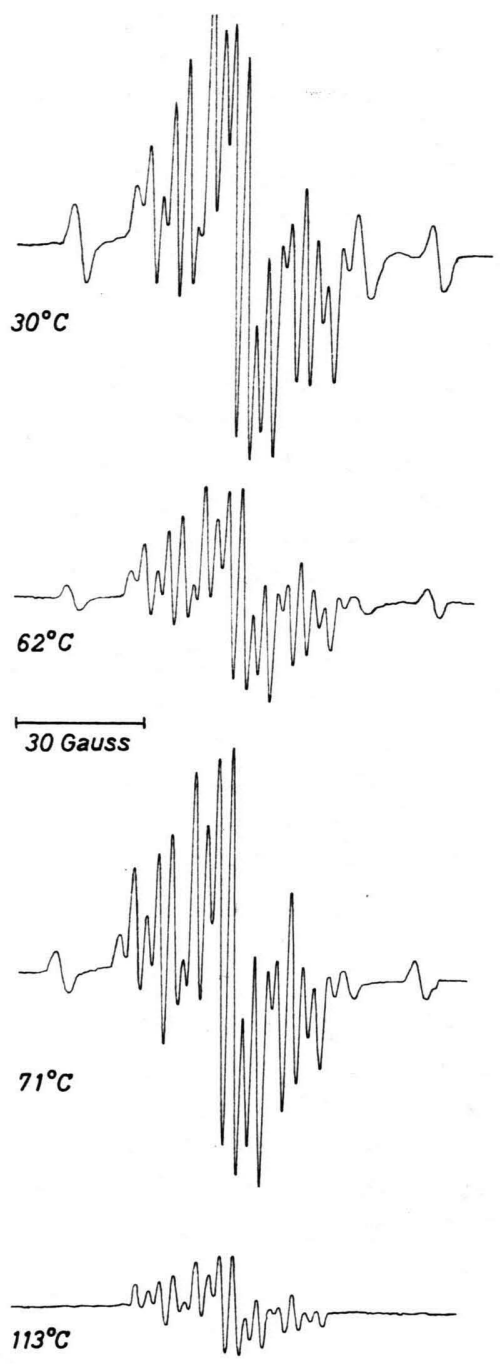

30 Gauss

Fig. 2. ESR spectra of a) $\mathrm{Co}^{60} \gamma$ a nd b) electron irradiated adamantane. Irradiation temperature $25^{\circ} \mathrm{C}$. Dose $=0.5 \mathrm{Mrad}$. Instrument gain varied according to signal intensity.

of the species produced. In contrast to Fig. 1 these signals show resolution more characteristic of radical species in solution, though the average line width is 1.6 gauss. No change in spectral width was observable in the temperature range $25^{\circ}-110^{\circ} \mathrm{C}$ where the last detectable signal was recorded. Adamantane prepared by recrystallization as described by FERRELL ${ }^{4}$ or by too rapidly zone-refining gave in no circumstances such well resolved spectra. Indeed the spectrum obtained from recrystallized adamantane $\gamma$-irradiated and recorded at room temperature strongly resembled those shown in Fig. 1 though much lower in intensity. That the spectra obtained from adamantane irradiated at room temperature consist of a mixture of radicals in unequal proportions is confirmed by the following observations i) the ratio of peak intensities of the outermost wing lines to that of a prominant line in the central part of the spectrum shows a variation of $1.70-3.0$ in the temperature range $30-70^{\circ} \mathrm{C}$, 
though the line width of the lines used remains constant.

ii) the central part of the spectrum remains distinct long after the disappearance of the outer lines. Particularly interesting in connection with the latter point however is that superposition of spectra before and after loss of the wing lines does not reveal any concurrent loss of lines or change in line width in the central part of the spectrum. iii) the disappearance of the outer lines is irreversible i.e. they do not reappear on cooling. We may therefore rule the possibility that we are dealing with a dynamically exchanging single species. It must be that the low temperature species is present in low concentration and/or has a broad low intensity absorption the loss of which has no considerable effect on the main spectrum. Our reasons for assigning the main component of the spectrum to the 1-adamantyl radical have been reported elsewhere ${ }^{9}$ and will therefore be only briefly repeated here. Whatever the credibility of the earlier work, we may safely say that i) the large triplet (46.7 gauss) observed at room temperature by GEE et al. was not noted by us. ii) the major doublet splitting (20.5 gauss) observed by FERRELL et al. ${ }^{4}$ at $-34{ }^{\circ} \mathrm{C}$ is also absent. iii) the remarkable difference in stability between our species and those of the other workers ${ }^{3,4}$ suggests radicals of a differing nature. iv) the fact that our spectrum is best analysed on the basis of a major triplet splitting of 14.28 gauss (adjacent $\mathrm{CH}_{2}$ group, see Table I).

Table I. Hyperfine coupling constants used in the analysis of the stable component of the adamantane spectrum ${ }^{9 a}$ (number of protons inbrackets).

\begin{tabular}{cccc}
\hline $\mathrm{a}_{1}$ & $\mathrm{a}_{2}$ & $\mathrm{a}_{3}$ & $\mathrm{a}_{4}$ \\
\hline $14.28(2)$ & $5.62(2)$ & $3.56(1)$ & $3.3(2)$ \\
$14.28(2)$ & $5.62(2)$ & $3.56(2)$ & $3.3(1)$ \\
\hline
\end{tabular}

The nature of the species giving rise to the wing lines can clearly not be adduced from the available evidence. We may rule out the 2-adamantyl radical however, since it probably has a spectrum of such complexity that its disappearance from a mixed spectrum would cause far more than the observed effect. We suggest therefore that it may be a smaller fragment disrupted from the adamantane molecule. That such fragments are formed during radiolysis of adamantane is confirmed by the observation of HATTENBACH and vom BAUR ${ }^{8}$ of $\mathrm{Cl}-\mathrm{C} 3$ hydro- carbons in the reaction products. The suggestion that such radicals are primary in nature is sup. ported by our observation that they are observed after only very small doses of irradiation $(\cong 20$ krads). Examination of Fig. 2 reveals no essential change in the nature of the main spectrum during warming, thus effectively excluding the possibility of radical isomerization ${ }^{3}$ in this range of temperature.

The reason for the remarkable influence of irradiation temperature on the radical stability ${ }^{*}$ is at the present time unclear though it is reminiscent of several other recent studies ${ }^{10-13}$ in which thermal treatment has been shown to play an important role in determining the nature and properties of radiation induced radicals. We are currently conducting further work aimed at elucidating the relevance of the transition point in controlling radical stability. $\mathrm{G}$ (radical) values and a knowledge of the nature of the decomposition kinetics around this temperature should give more evidence about the processes involved.

The mechanism of formation of radicals from molecules trapped in the adamantane matrix

Radiolysis of previously recrystallized adamantane has been shown to yield radicals derived exclusively from solvent molecules included in the solid matrix ${ }^{5-7}$. The stability of such trapped radicals makes them especially amenable to detailed ESR investigation. WOOD and LLOYD ${ }^{5}$, noting the specificity ${ }^{* *}$ with which these radicals are produced suggested that energy, possibly by an exciton mechanism, is transferred directly from the adamantane to the included molecules. The fact that the radicals formed are always those resulting from hydrogen abstraction from the guest molecule was explained by assuming a more dominant cage effect operating against diffusion of larger fragments.

We believe that a more satisfactory mechanism can be arrived at by considering the following factors a) though the electron fractions for adamantane

\footnotetext{
* We have separate evidence that the radicals produced by irradiation at $77^{\circ} \mathrm{K}$ and warming to room temperature are identical with those found by irradiation at the latter temperature. This effect was noted for both electron and $\gamma$ irradiation.

** for example, primary amines form only one aminoalkyl radical and then only if they have an $a$ hydrogen atom. Non symmetrical secondary and tertiary amines similarly give only a single species ${ }^{5}$.
} 
and smaller organic substrates will always be approximately equal, the weight fraction of adamantane will always dominate. The direct action of radiation can therefore be ignored, b) the sensitivity of adamantane to radiation as noted by HATTEN$\mathrm{BACH}^{8}$ and ourselves, c) our observation of the 1-adamantyl radical in pure irradiated adamantane, d) the expectation that the former would have properties in common with other sterically hindered radicals such as tert-butyl or tert-butoxy. We imagine, therefore the following processes to occur during the production of "guest" radicals. High energy irradiation produces a high concentration of radicals distributed within the adamantane matrix. At room temperature these predominantly 1-adamantyl radicals are extremely stable in the absence of foreign material. In the presence of suitably situated guest molecules however they may take part reactions leading to the trapped. radicals $\mathrm{S}$, (see below)

$$
\begin{array}{r}
\mathrm{C}_{10} \mathrm{H}_{16} \stackrel{\gamma \text { or e }}{\longrightarrow} \mathrm{C}_{10} \mathrm{H}_{16}{ }^{+\cdot}+\mathrm{e} \rightarrow \mathrm{C}_{10} \mathrm{H}_{15}+\mathrm{H}^{+} \rightarrow \text { products } \\
\text { (slow in absence of foreign material) } \\
\mathrm{C}_{10} \mathrm{H}_{15}{ }^{+}+\mathrm{SH} \rightarrow \mathrm{C}_{10} \mathrm{H}_{16}+\mathrm{S}^{\cdot}
\end{array}
$$

Two points are favour such a mechanism. Firstly in the case of the unsymmetrical amines presented by WOOD and LLOYD the radicals are more often than not products of $\mathrm{H}$ abstraction from the least

1 C. E. Nordman and D. L. Schmitkons, Acta crystallogr. 18, 76A [1965].

2 D. W. McCall and D. C. Douglas, J. chem. Physics 33, 777 [1960]

3 D. R. Gee, L. Fabes, and J. K. S. WanN, Chem. Phys. Letters 7, 311 [1970].

4 J. R. Ferrell, G. R. Holden, JR., R. V. Lloyd, and D. E. Wood, Chem. Phys. Letters 9, 343 [1971].

5 D. E. WOod and R. V. Lloyd, J. chem. Physics 53, 3932 [1970].

6 R. V. Lloyd and D. E. Wood, Molecular Physics 20, 735 [1971].

7 J. E. Bennett and B. Mile, Trans. Faraday Soc. 67, 1587 [1971].

8 K. Hattenbach and E. vom Bauer, Atomkernenergie 11, 262 [1962]. hindered side chain. Secondly, the well established property of hindered radicals to preferentially undergo such reactions ${ }^{15}$. Certainly the former point is more easily explicable on the basis of steric effects than on the specific energy transfer mechanism presented earlier. We see therefore that two main conditions must be fulfilled for the observation of trapped radicals in adamantane $e . g$. a) a sufficiently high concentration of substrate must be present in order that the adamantane and substrate reaction will be favoured, b) the reaction must present an advantageous pathway for decay of the adamantyl radicals. Point (b) will clearly become of importance when the hydrogen atom to be abstracted is unfavourably placed i.e. sterically hindered, for in this case the competition between adamantyl radical recombination and hydrogen atom abstraction will be displaced in favour of the former. The failure of WOOD and LLOYD to observe radicals from tert-butyl-amine may well be connected with this latter point since reaction ( $\mathrm{H}$ abstraction) of 1-adamantyl with the tert-butyl group would presumably be almost as sterically unfavourable as recombination of the 1-adamantyl radicals themselves.

The authors wish to express their thanks to Mrs. D. RöHM for the gas chromatographic analyses and Mr. D. RUDOLPH for operation of the Linac electron accelerator.

9 a) W. G. Filby and K. Günther, Chem. Phys. Letters 4, 440 [1972] ; b) W. G. Filby and K. GüNTHER, ibid., submitted for publication.

10 H. C. Box, H. G. Freund, and E. E. Budzinski, J. chem. Physics 46, 4470 [1967].

11 J. W. Sinclair and M. A. Hanna, J. physic. Chem. 71, 84 [1967].

12 M. T. Rogers and L. D. Kispert, Radiation Chemistry II, Adv. in Chem. Series, 327 [1968].

13 V. K. Ermolaev and V. V. Voevodsky, Proc. sec. Tihany Symp. Rad. Chem. 211 [1967].

14 L. Bonnazola and R. Marx, Chem. Phys. Letters 8, 413 [1971].

15 W. A. Pryor, "Free Radicals", McGraw-Hill Book Co., New York 1966 